\title{
Towards establishing the second $b$-flavored CKM unitarity triangle
}

\author{
Zhi-zhong Xing* and Di Zhang ${ }^{1}$ \\ Institute of High Energy Physics and School of Physical Sciences, University of Chinese Academy of \\ Sciences, \\ Beijing 100049, China \\ E-mail: xingzz@ihep.ac.cn, zhangdi@ihep.ac.cn
}

Some fine differences between the twin $b$-flavored unitarity triangles are calculated by means of a generalized Wolfenstein parametrization of the CKM matrix, and a possibility of experimentally establishing the second triangle is briefly discussed. We find that the apexes of these two triangles, characterized respectively by $(\bar{\rho}, \bar{\eta})$ and $(\widetilde{\rho}, \widetilde{\eta})$, are located on the same circular arc in the complex plane. This observation provides us with a new way to test consistency of the CKM picture of $\mathrm{CP}$ violation in the quark sector and probe possible new physics. The differences between the apexes (i.e., $\widetilde{\rho}-\bar{\rho}$ and $\widetilde{\eta}-\bar{\eta}$ ) are found to be of $O\left(\lambda^{2}\right)$ with $\lambda \simeq 0.22$ being the Wolfenstein expansion parameter, and the shapes of these two triangles are found to be insensitive to the two-loop renormalization-group-equation running effects up to the accuracy of $O\left(\lambda^{4}\right)$.

40th International Conference on High Energy physics - ICHEP2020

July 28 - August 6, 2020

Prague, Czech Republic (virtual meeting)

${ }^{1}$ Corresponding author

* Speaker 


\section{Introduction}

In the standard model (SM) the quark fields interact with both the gauge fields and the Higgs field, leading to a nontrivial mismatch between the flavor and mass eigenstates of quarks. This kind of mismatch, which is described by the $3 \times 3$ Cabibbo-Kobayashi-Maskawa (CKM) matrix $V[1,2]$, implies the existence of flavor mixing and $\mathrm{CP}$ violation. The unitarity of $V$ is strictly guaranteed by the SM itself, and it can be geometrically described by six unitarity triangles in the complex plane. In the past twenty years, the $b$-flavored unitarity triangle $\Delta_{s}$, defined by the orthogonality relation $V_{u b}^{*} V_{u d}+V_{c b}^{*} V_{c d}+V_{t b}^{*} V_{t d}=0$, has been extensively studied. In fact, this triangle has played a significant role in verifying the success of the Kobayashi-Maskawa mechanism of CP violation [2]. On the contrary, the other $b$-flavored triangle $\Delta_{c}$ (defined by $V_{u d}^{*} V_{t d}+V_{u s}^{*} V_{t s}+V_{u b}^{*} V_{t b}=0$ in the complex plane) has largely been ignored. The rescaled versions of $\Delta_{s}$ and $\Delta_{c}$, denoted respectively as

$$
\Delta_{s}^{\prime}: 1+\frac{V_{u b}^{*} V_{u d}}{V_{c b}^{*} V_{c d}}+\frac{V_{t b}^{*} V_{t d}}{V_{c b}^{*} V_{c d}}=0, \quad \Delta_{c}^{\prime}: 1+\frac{V_{u d}^{*} V_{t d}}{V_{u s}^{*} V_{t s}}+\frac{V_{u b}^{*} V_{t b}}{V_{u s}^{*} V_{t s}}=0,
$$

are highly similar in shape, as illustrated in Figure 1. Hence they are referred to as the twin $b$-flavored unitarity triangles [3]. The apexes of triangles $\Delta_{s}^{\prime}$ and $\Delta_{c}^{\prime}$ are defined as

$$
\bar{\rho}+\mathrm{i} \bar{\eta}=-\frac{V_{u b}^{*} V_{u d}}{V_{c b}^{*} V_{c d}}, \quad \widetilde{\rho}+\mathrm{i} \widetilde{\eta}=-\frac{V_{u b}^{*} V_{t b}}{V_{u s}^{*} V_{t s}}
$$

in the complex plane, respectively. An intriguing question is whether these two twin triangles can be distinguished by the upcoming higher-precision measurements to be carried out at the super- $B$ factory [4] and the high-luminosity Large Hadron Collider (LHC) [5, 6].

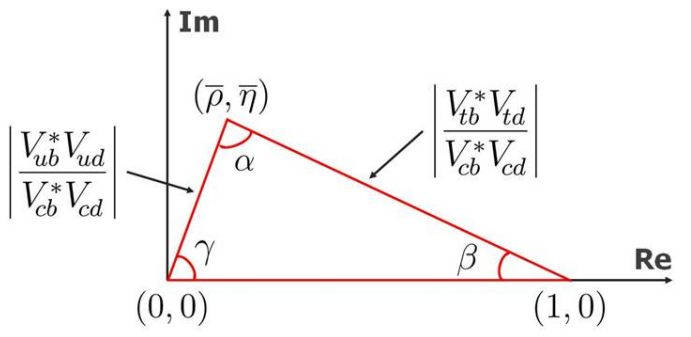

(a)

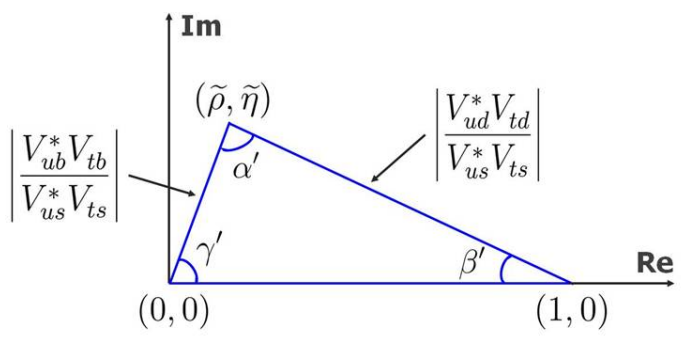

(b)

Figure 1: An illustration of the twin $b$-flavored unitarity triangles $\Delta_{s}^{\prime}$ and $\Delta_{c}^{\prime}$ in the complex plane, where $(\bar{\rho}, \bar{\eta})$ and $(\widetilde{\rho}, \widetilde{\eta})$ denote their apexes, respectively.

In this talk we report our recent study of fine differences between triangles $\Delta_{s}^{\prime}$ and $\Delta_{c}^{\prime}$ [3]. We find that their apexes are actually located on a circular arc in the complex plane. To experimentally distinguish $\triangle_{S}^{\prime}$ and $\triangle_{c}^{\prime}$ at the $3 \sigma$ level, the apexes should be measured to the precision of $\lesssim 0.4 \%$. A possible way to separately establish $\triangle_{s}^{\prime}$ and $\triangle_{c}^{\prime}$ is to use the experimental data from $B_{u}^{ \pm}$and $B_{d}^{0}-\bar{B}_{d}^{0}$ systems and those from $B_{u}^{ \pm}$and $B_{s}^{0}-\bar{B}_{s}^{0}$ systems, respectively. In addition, we find that the apexes and inner angles of $\Delta_{s}^{\prime}$ and $\Delta_{c}^{\prime}$ are insensitive to the two-loop renormalization-group-equation (RGE) evolution up to the accuracy of $O\left(\lambda^{4}\right)$ with $\lambda \simeq 0.22$. 


\section{The apexes of $\triangle_{s}^{\prime}$ and $\Delta_{c}^{\prime}$ on a circular arc}

A popular extension of the original Wolfenstein parametrization [7] of the CKM matrix $V$ is $[8,9]$

$$
V \simeq\left(\begin{array}{ccc}
1-\frac{1}{2} \lambda^{2}-\frac{1}{8} \lambda^{4} & \lambda & A \lambda^{3}(\rho-\mathrm{i} \eta) \\
-\lambda+\frac{1}{2} A^{2} \lambda^{5}[1-2(\rho+\mathrm{i} \eta)] & 1-\frac{1}{2} \lambda^{2}-\frac{1}{8} \lambda^{4}\left(1+4 A^{2}\right) & A \lambda^{2} \\
A \lambda^{3}(1-\rho-\mathrm{i} \eta)+\frac{1}{2} A \lambda^{5}(\rho+\mathrm{i} \eta) & -A \lambda^{2}+\frac{1}{2} A \lambda^{4}[1-2(\rho+\mathrm{i} \eta)] & 1-\frac{1}{2} A^{2} \lambda^{4}
\end{array}\right)
$$

up to the accuracy of $O\left(\lambda^{6}\right)$. Note that here $V_{u b} \equiv A \lambda^{3}(\rho-\mathrm{i} \eta)$ is exact by definition. Moreover, the exact relationship between $(\rho, \eta)$ and $(\widetilde{\rho}, \widetilde{\eta})$ or $(\bar{\rho}, \bar{\eta})$ is given by

$$
\rho+\mathrm{i} \eta=\frac{\sqrt{1-A^{2} \lambda^{4}}(\bar{\rho}+\mathrm{i} \bar{\eta})}{\sqrt{1-\lambda^{2}}\left[1-A^{2} \lambda^{4}(\bar{\rho}+\mathrm{i} \bar{\eta})\right]}=\frac{\sqrt{1-\lambda^{2}}(\widetilde{\rho}+\mathrm{i} \widetilde{\eta})}{\sqrt{1-A^{2} \lambda^{4}}\left[1-\lambda^{2}(\widetilde{\rho}+\mathrm{i} \widetilde{\eta})\right]} .
$$

If both the apexes $(\widetilde{\rho}, \widetilde{\eta})$ and $(\bar{\rho}, \bar{\eta})$ can be directly determined from some precision measurements, a comparison between the results of $(\rho, \eta)$ obtained independently from Eq. (4) will provide a meaningful consistency check of the CKM picture for CP violation described by $\Delta_{s}^{\prime}$ and $\Delta_{c}^{\prime}$ in the SM. Unfortunately, so far no effort has been made towards establishing $\triangle_{c}^{\prime}$ from the available experimental data. It is well known that the apex of $\Delta_{s}^{\prime}$ has been excessively constrained by current experimental results for $\left|V_{u b}\right| /\left|V_{c b}\right|, \sin 2 \beta$ (CP violation in $B_{d}^{0}$ vs $\bar{B}_{d}^{0} \rightarrow J / \psi K_{\mathrm{S}}$ decays), $\Delta m_{d}$ (the mass difference of $B_{d}^{0}-\bar{B}_{d}^{0}$ mixing), $\Delta m_{s}$ (the mass difference of $B_{s}^{0}-\bar{B}_{s}^{0}$ mixing), $\varepsilon_{K}$ (CP violation in $K^{0}-\bar{K}^{0}$ mixing) and so on [9-11]. To separately constrain the apexes of $\Delta_{s}^{\prime}$ and $\Delta_{c}^{\prime}$, one may make use of the experimental data from $B_{u}^{ \pm}$and $B_{d}^{0}-\bar{B}_{d}^{0}$ systems and those from $B_{u}^{ \pm}$and $B_{s}^{0}-\bar{B}_{s}^{0}$ systems, respectively. The measurements of $\Delta m_{d}$ and $\Delta m_{s}$, which depend respectively on $V_{t b}^{*} V_{t d}$ and $V_{t b}^{*} V_{t s}$ via the $t$-dominated box diagrams, are expected to be useful to distinguish between the apexes of $\triangle_{s}^{\prime}$ and $\triangle_{c}^{\prime}$. It is also possible to determine $\left|V_{t b}^{*} V_{t s}\right|$ from more precise measurements of $B \rightarrow X_{s} \gamma$ and $B_{s} \rightarrow \mu^{+} \mu^{-}$decays in the near future [10].

Therefore, in the present case, we just calculate $(\rho, \eta)$ and then $(\widetilde{\rho}, \widetilde{\eta})$ by means of of Eq. (4) with $\lambda=0.22453 \pm 0.00044, A=0.836 \pm 0.015, \bar{\rho}=0.122_{-0.017}^{+0.018}$ and $\bar{\eta}=0.355_{-0.011}^{+0.012}$ [10]. We obtain $\rho=0.125 \pm 0.018$ and $\eta=0.364 \pm 0.012$, together with $\widetilde{\rho}=0.134 \pm 0.018$ and $\widetilde{\eta}=0.368 \pm 0.012$. Taking advantage of Eqs. (2) and (3), we directly arrive at

$$
\widetilde{\rho}-\bar{\rho} \simeq\left[\rho(1-\rho)+\eta^{2}\right] \lambda^{2}, \quad \tilde{\eta}-\bar{\eta} \simeq \eta(1-2 \rho) \lambda^{2},
$$

up to the accuracy of $O\left(\lambda^{4}\right)$. So the differences $\widetilde{\rho}-\bar{\rho}$ and $\widetilde{\eta}-\bar{\eta}$ remain within the error bars of these four parameters. We expect that $\Delta_{c}^{\prime}$ and $\Delta_{s}^{\prime}$ will be experimentally distinguishable at the $3 \sigma$ level if their apexes $(\bar{\rho}, \bar{\eta})$ and $(\widetilde{\rho}, \widetilde{\eta})$ can be determined to the precision of $\lesssim 0.4 \%$.

Let us proceed to take a look at the inner angles of triangles $\Delta_{s}^{\prime}$ and $\Delta_{c}^{\prime}$, which are defined as

$$
\begin{array}{rlr}
\alpha \equiv \arg \left(-\frac{V_{t b}^{*} V_{t d}}{V_{u b}^{*} V_{u d}}\right), & \beta \equiv \arg \left(-\frac{V_{c b}^{*} V_{c d}}{V_{t b}^{*} V_{t d}}\right), & \gamma \equiv \arg \left(-\frac{V_{u b}^{*} V_{u d}}{V_{c b}^{*} V_{c d}}\right), \\
\alpha^{\prime} \equiv \arg \left(-\frac{V_{u d}^{*} V_{t d}}{V_{u b}^{*} V_{t b}}\right), & \beta^{\prime} \equiv \arg \left(-\frac{V_{u s}^{*} V_{t s}}{V_{u d}^{*} V_{t d}}\right), & \gamma^{\prime} \equiv \arg \left(-\frac{V_{u b}^{*} V_{t b}}{V_{u s}^{*} V_{t s}}\right) .
\end{array}
$$




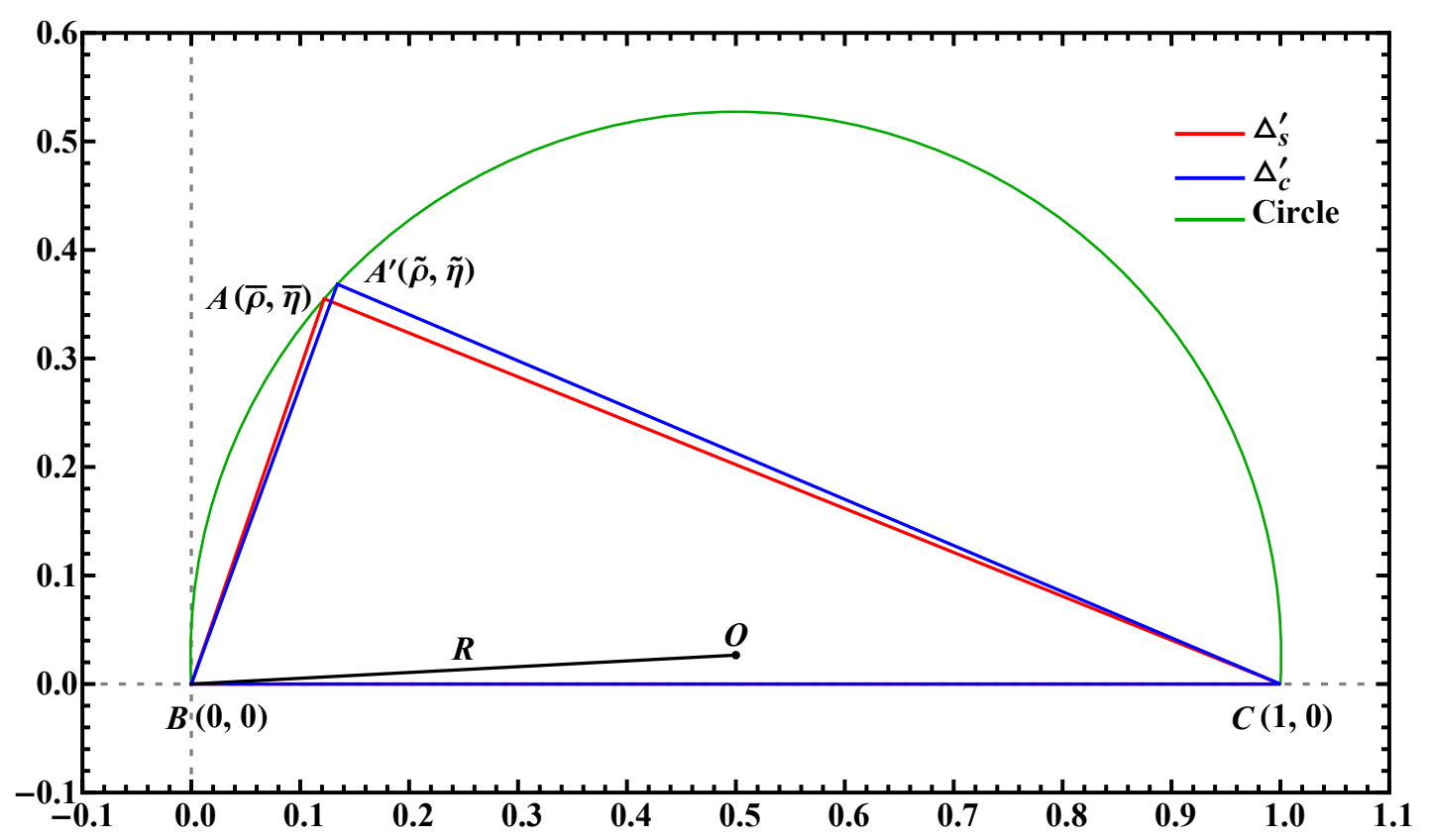

Figure 2: The rescaled CKM unitarity triangles $\triangle_{S}^{\prime}=\triangle A B C$ and $\triangle_{c}^{\prime}=\triangle A^{\prime} B C$ in the complex plane, where the center and radius of the circular arc are $O=(0.5,0.5 \cot \alpha)$ and $R=0.5 \csc \alpha$.

With the help of Eqs. (3) and (6), one can achieve that $\alpha^{\prime}=\alpha$ holds exactly by definition, and

$$
\beta^{\prime}-\beta=\gamma-\gamma^{\prime} \simeq \eta \lambda^{2}\left[1+\left(\frac{1}{2}-A^{2}-\rho\right) \lambda^{2}\right]
$$

Taking account of the values of $\lambda, A, \bar{\rho}$ and $\bar{\eta}$, we immediately obtain $\alpha=\alpha^{\prime} \simeq 87.0^{\circ} \pm 2.5^{\circ}$, $\beta \simeq 22.0^{\circ} \pm 0.8^{\circ}, \gamma \simeq 71.0^{\circ} \pm 2.6^{\circ}, \beta^{\prime} \simeq 23.0^{\circ} \pm 0.8^{\circ}$ and $\gamma^{\prime} \simeq 70.0^{\circ} \pm 2.6^{\circ}$. So the numerical results of $\beta^{\prime}-\beta$ and $\gamma-\gamma^{\prime}$, which also characterize the tiny difference between triangles $\triangle_{s}^{\prime}$ and $\Delta_{c}^{\prime}$, remain within the error bars of these four angles and hence require more accurate measurements.

Now that the twin rescaled unitarity triangles $\Delta_{c}^{\prime}$ and $\Delta_{S}^{\prime}$ share a common inner angle $\alpha^{\prime}=\alpha$ and a common side $B C$ as shown in Figure 2, their corresponding apexes $(\widetilde{\rho}, \widetilde{\eta})$ and $(\bar{\rho}, \bar{\eta})$ must be located on a circular arc in the upper complex plane. That is

$$
\left(\widetilde{\rho}-\frac{1}{2}\right)^{2}+\left(\widetilde{\eta}-\frac{1}{2} \cot \alpha\right)^{2}=\left(\bar{\rho}-\frac{1}{2}\right)^{2}+\left(\bar{\eta}-\frac{1}{2} \cot \alpha\right)^{2}=\left(\frac{1}{2} \csc \alpha\right)^{2} .
$$

It is obvious that the center and radius of the circular arc determined by Eq. (8) and shown in Figure 2 are $O=(0.5,0.5 \cot \alpha)$ and $R=0.5 \csc \alpha$. The fact that all the apexes of $\Delta_{c}^{\prime}$ and $\Delta_{s}^{\prime}$ are located on the same circular arc is of course a natural consequence of the CKM unitarity. It provides another interesting way to test the consistency of quark flavor mixing and CP violation in the SM. Since $\triangle_{S}^{\prime}$ has been established to a very good degree of accuracy, it allows us to fix a benchmark circular arc as shown in Figure 2. The future measurements of $(\widetilde{\rho}, \widetilde{\eta})$ will tell us to what extent the experimental values of this apex are also located on the same circular arc. In other words, an experimental test of the equality given in Eq. (8) will be greatly useful at both the super- $B$ factory and the high-luminosity LHC. 


\section{Two-loop RGE evolution of $\triangle_{s}^{\prime}$ and $\triangle_{c}^{\prime}$}

Note that elements of the CKM matrix $V$ depend on the energy scale $\Lambda$. When $\Lambda$ is far above the electroweak scale $\Lambda_{\mathrm{EW}} \sim 10^{2} \mathrm{GeV}$, the RGE running effects of $V$ will become appreciable and should be taken into account. In particular, the two-loop RGEs of $V$ have been derived in the framework of the SM or its minimal supersymmetric version (MSSM) [12-15]. In view of $y_{u} / y_{c} \sim y_{c} / y_{t} \sim \lambda^{4}, y_{d} / y_{s} \sim y_{s} / y_{b} \sim \lambda^{2}$ at a given energy scale and the relatively strong hierarchies of those off-diagonal elements of $V$, Barger et al have found [14]

$$
\frac{\mathrm{d}}{\mathrm{d} t}\left(\begin{array}{ccc}
\left|V_{u d}\right| & \left|V_{u s}\right| & \left|V_{u b}\right| \\
\left|V_{c d}\right| & \left|V_{c s}\right| & \left|V_{c b}\right| \\
\left|V_{t d}\right| & \left|V_{t s}\right| & \left|V_{t b}\right|
\end{array}\right) \simeq\left(S_{1}+S_{2}\right)\left(\begin{array}{ccc}
0 & 0 & \left|V_{u b}\right| \\
0 & 0 & \left|V_{c b}\right| \\
\left|V_{t d}\right| & \left|V_{t s}\right| & 0
\end{array}\right), \quad \frac{\mathrm{d} \mathcal{J}}{\mathrm{d} t} \simeq 2\left(S_{1}+S_{2}\right) \mathcal{J},
$$

where $t \equiv \ln \left(\Lambda / \Lambda_{\mathrm{EW}}\right), \mathcal{J} \equiv \operatorname{Im}\left(V_{u d} V_{c s} V_{u s}^{*} V_{c d}^{*}\right) \simeq A^{2} \lambda^{6} \eta$ is the Jarlskog invariant of CP violation [16], $S_{1}$ and $S_{2}$ stand respectively for the one- and two-loop contributions to the RGEs of $V$ (and their explicit definitions can be found in Ref. [3]).

After a careful check of the approximations made in obtaining Eq. (9), we conclude that the two-loop RGEs shown in Eq. (9) are valid up to the accuracy of $O\left(\lambda^{4}\right)$. To the same order, the integral solutions of the Wolfenstein parameters to Eq. (9) can be figured out as follows:

$$
\begin{aligned}
& \lambda(\Lambda) \simeq \lambda\left(\Lambda_{\mathrm{EW}}\right), \quad \rho(\Lambda) \simeq \rho\left(\Lambda_{\mathrm{EW}}\right), \quad \eta(\Lambda) \simeq \eta\left(\Lambda_{\mathrm{EW}}\right), \quad A(\Lambda) \simeq I_{1} I_{2} A\left(\Lambda_{\mathrm{EW}}\right) ; \\
& \bar{\rho}(\Lambda) \simeq \bar{\rho}\left(\Lambda_{\mathrm{EW}}\right), \quad \bar{\eta}(\Lambda) \simeq \bar{\eta}\left(\Lambda_{\mathrm{EW}}\right), \quad \widetilde{\rho}(\Lambda) \simeq \widetilde{\rho}\left(\Lambda_{\mathrm{EW}}\right), \quad \widetilde{\eta}(\Lambda) \simeq \widetilde{\eta}\left(\Lambda_{\mathrm{EW}}\right),
\end{aligned}
$$

where $\Lambda$ denotes an arbitrary energy scale between $\Lambda_{\mathrm{EW}}$ and $\Lambda_{\mathrm{GUT}}$, and the loop functions $I_{1}$ and $I_{2}$ have also been defined in Ref. [3]. It is clear that $\left|V_{u b}\right|,\left|V_{c b}\right|,\left|V_{t d}\right|$ and $\left|V_{t s}\right|$ evolve in the same way as $A$, and $\mathcal{J}(\Lambda) \simeq I_{1}^{2} I_{2}^{2} \mathcal{J}\left(\Lambda_{\mathrm{EW}}\right)$ holds for the Jarlskog invariant. In comparison, $\left|V_{u d}\right|,\left|V_{u s}\right|$, $\left|V_{c d}\right|,\left|V_{c s}\right|$ and $\left|V_{t b}\right|$ are essentially stable against changes of the energy scale $\Lambda$. Thus the rescaled unitarity triangles $\Delta_{s}^{\prime}$ and $\Delta_{c}^{\prime}$ keep unchanged when the energy scale $\Lambda$ evolves from $\Lambda_{\mathrm{EW}}$ to $\Lambda_{\mathrm{GUT}}$ or vice versa, up to the accuracy of $O\left(\lambda^{4}\right)$. In other words, the overall shape of either of $\Delta_{s}$ and $\Delta_{c}$ keeps undeformed up to the same accuracy.

In summary, we have discussed whether the twin $b$-flavored unitarity triangles $\Delta_{s}^{\prime}$ and $\Delta_{c}^{\prime}$ can be experimentally distinguished from each other in the context of the upcoming precision measurements at the super- $B$ factory and the high-luminosity LHC. The answer is affirmative if their apexes are measured to a sufficiently good degree of accuracy. We have pointed out that the apexes $(\bar{\rho}, \bar{\eta})$ and $(\widetilde{\rho}, \widetilde{\eta})$ are exactly located on a circular arc, whose center and radius are $O=(0.5,0.5 \cot \alpha)$ and $R=0.5 \csc \alpha$ respectively. We have also shown that $(\bar{\rho}, \bar{\eta})$ and $(\widetilde{\rho}, \widetilde{\eta})$ are insensitive to the two-loop RGE running effects up to the accuracy of $O\left(\lambda^{4}\right)$. So the experimental results of all the inner angles of $\Delta_{s}^{\prime}$ and $\Delta_{c}^{\prime}$ obtained at low energies can directly be confronted with some theoretical predictions at a superhigh energy scale.

This work was supported in part by the National Natural Science Foundation of China under grant No. 11775231, grant No. 11835013 and grant No. 12075254.

\section{References}

[1] N. Cabibbo, Unitary Symmetry and Leptonic Decays, Phys. Rev. Lett. 10 (1963) 531. 
[2] M. Kobayashi and T. Maskawa, CP Violation in the Renormalizable Theory of Weak Interaction, Prog. Theor. Phys. 49 (1973) 652.

[3] Z. Z. Xing and D. Zhang, Distinguishing between the twin b-flavored unitarity triangles on a circular arc, Phys. Lett. B 803 (2020), 135302 [arXiv:1911.03292 [hep-ph]].

[4] E. Kou et al. [Belle-II Collaboration], The Belle II Physics Book, arXiv:1808.10567 [hep-ex].

[5] M. Bona et al. [SuperB Collaboration], SuperB: A High-Luminosity Asymmetric $e^{+} e^{-}$Super Flavor Factory. Conceptual Design Report, arXiv:0709.0451 [hep-ex].

[6] R. Aaij et al. [LHCb Collaboration], Physics case for an LHCb Upgrade II - Opportunities in flavour physics, and beyond, in the HL-LHC era, arXiv:1808.08865.

[7] L. Wolfenstein, Parametrization of the Kobayashi-Maskawa Matrix, Phys. Rev. Lett. 51 (1983) 1945.

[8] A. J. Buras, M. E. Lautenbacher and G. Ostermaier, Waiting for the top quark mass, $K^{+} \rightarrow$ $\pi^{+} v \bar{v}, B_{s}^{0}-\bar{B}_{s}^{0}$ mixing and CP asymmetries in B decays, Phys. Rev. D 50 (1994) 3433 [hep$\mathrm{ph} / 9403384]$.

[9] J. Charles et al. [CKMfitter Group], CP violation and the CKM matrix: Assessing the impact of the asymmetric B factories, Eur. Phys. J. C 41 (2005) 1 [hep-ph/0406184].

[10] M. Tanabashi et al. [Particle Data Group], Review of Particle Physics, Phys. Rev. D 98, no. 3, 030001 (2018).

[11] M. Bona et al. [UTfit Collaboration], The 2004 UTfit collaboration report on the status of the unitarity triangle in the standard model, JHEP 0507 (2005) 028 [hep-ph/0501199].

[12] M. E. Machacek and M. T. Vaughn, Two Loop Renormalization Group Equations in a General Quantum Field Theory. 2. Yukawa Couplings, Nucl. Phys. B 236 (1984) 221.

[13] V. D. Barger, M. S. Berger and P. Ohmann, Supersymmetric grand unified theories: Two loop evolution of gauge and Yukawa couplings, Phys. Rev. D 47 (1993) 1093 [hep-ph/9209232].

[14] V. D. Barger, M. S. Berger and P. Ohmann, Universal evolution of CKM matrix elements, Phys. Rev. D 47 (1993) 2038 [hep-ph/9210260].

[15] M. x. Luo and Y. Xiao, Two loop renormalization group equations in the standard model, Phys. Rev. Lett. 90 (2003) 011601 [hep-ph/0207271].

[16] C. Jarlskog, Commutator of the Quark Mass Matrices in the Standard Electroweak Model and a Measure of Maximal CP Violation, Phys. Rev. Lett. 55 (1985) 1039. 